\title{
APPLICATION AND MODIFICATION OF GODA FORMULAE FOR NONIMPULSIVE WAVE FORCES ON ELEVATED COASTAL STRUCTURES
}

\author{
Tori Tomiczek, United States Naval Academy, toritomi11@gmail.com \\ Amy Wyman, Oregon State University, wymana@oregonstate.edu \\ Hyoungsu Park, Oregon State University, Hyoungsu.Park@gmail.com \\ Daniel T. Cox, Oregon State University, dan.cox@oregonstate.edu
}

\section{IMPORTANCE}

With an increase in both the population densities in coastal communities and the frequency of high-intensity tropical cyclones in recent years, effective strategies to mitigate wave and surge-induced structural damage are essential to ensure community resilience. To prevent damage in vulnerable areas, structures must be constructed or retrofitted to withstand the wave and surge induced forces associated with extreme events. Accurately predicting the pressure distributions and forces caused by hurricane waves is a critical first step in establishing reliable design standards and construction practices. While progress has been made in the estimation of wave-induced horizontal loads on vertical walls and breakwaters or vertical loads on elevated slabs based on previous theoretical, experimental, and numerical investigations, the results of these studies may not directly apply to the geometries of coastal houses, which comprise a major portion of the building stock in coastal communities. Many coastal residences are elevated above ground; thus, methods must be developed that can predict both the horizontal and the vertical forces acting on the structure. This work uses results from a large scale experimental dataset to develop a robust methodology for estimating the waveinduced horizontal and vertical pressure distributions on an elevated structure.

\section{PHYSICAL MODEL AND COMPARISON WITH GODA} (2010) WAVE PRESSURE FORMULAE

A large (1:10) scale physical model was constructed in Oregon State University's Large Wave Flume (LWF) based on an idealization of an elevated coastal house on the Bolivar Peninsula, TX, USA, that was affected by surge and waves during Hurricane lke (2008). The specimen (Fig. 1) was installed with the capability to vary its elevation within the flume. Regular and irregular wave trials varied wave height and period while holding water depth constant; pressure transducers and load cells recorded the horizontal and vertical pressures and forces on the specimen at each structural elevation and wave condition. Table 1 Lists the wave conditions tested that produced nonimpulsive wave interactions with the elevated structure.
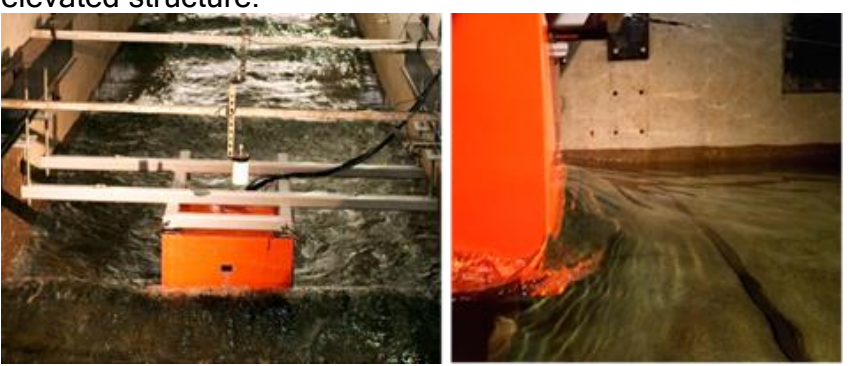

Figure 1 - Nonbreaking wave impacting test specimen: front (left panel) and side views (right panel).
Table 1- Experimental Conditions

\begin{tabular}{ccccccc}
\hline Exp & REG & & & \multicolumn{3}{c}{ TMA } \\
\hline & $\bar{H}(\mathrm{~m})$ & $\bar{T}(\mathrm{~s})$ & Dur.(min) & $H_{1 / 3}(\mathrm{~m})$ & $T_{p}(\mathrm{~s})$ & Dur.(min) \\
\hline X1 & 0.10 & 4.10 & 4.00 & 0.09 & 3.72 & 40.0 \\
X2 & 0.23 & 4.10 & 4.00 & 0.20 & 3.86 & 40.0 \\
X6 & 0.15 & 2.52 & 2.50 & 0.14 & 2.52 & 25.0 \\
\hline
\end{tabular}

The average pressure distributions at the times of the maximum horizontal and vertical forces were determined for each trial: an example is shown in Fig. 2 for a trial with the specimen's base positioned at the still water level. For each nonbreaking wave height-structural air gap condition, measured pressure horizontal and vertical pressure distributions were integrated over the area of impact to determine the experimental horizontal and vertical forces on the elevated structure.
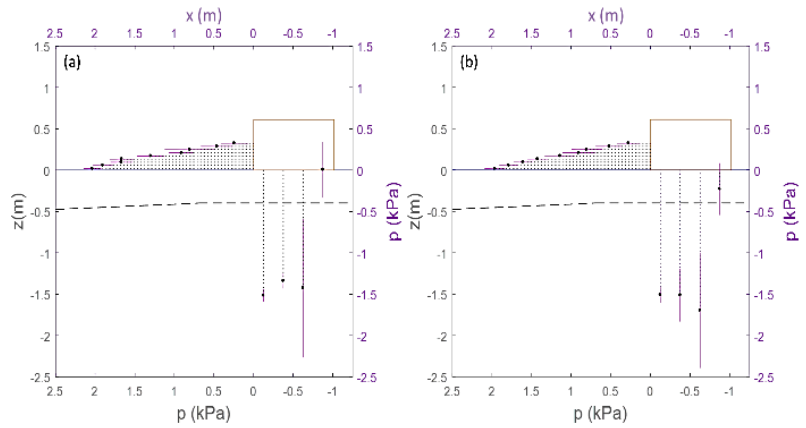

Figure 2 - Ensemble averaged horizontal and vertical pressure distributions showing $90 \%$ confidence intervals (magenta lines) at the time of the maximum (a) horizontal and (b) vertical forces. Horizontal and vertical pressures are plotted with respect to the bottom and right axes, and distance from the leading edge of the specimen and elevation above the still water are plotted on the top and left axes. The dashed line indicates the flume bathymetry.

These experimental forces were then compared with the forces obtained by integrating the pressure distribution calculated using the well-known method of Goda (2010), which was modified for elevated structures by Wiebe et al. (2014). Figure 3 shows two scenarios of the Wiebe et al. (2014) equations applied to an elevated structure: the first scenario shows a structure with lowest horizontal member above the stillwater level (positive air gap) and the second scenario shows the structure with a negative air gap being impacted by impacted by a wave. The equations to calculate defining parameters $\left(\eta^{*}, p_{1}, p_{5}, p_{3}\right)$ for the pressure distribution shown in Fig. 3 are described in Wiebe et al. (2014). 


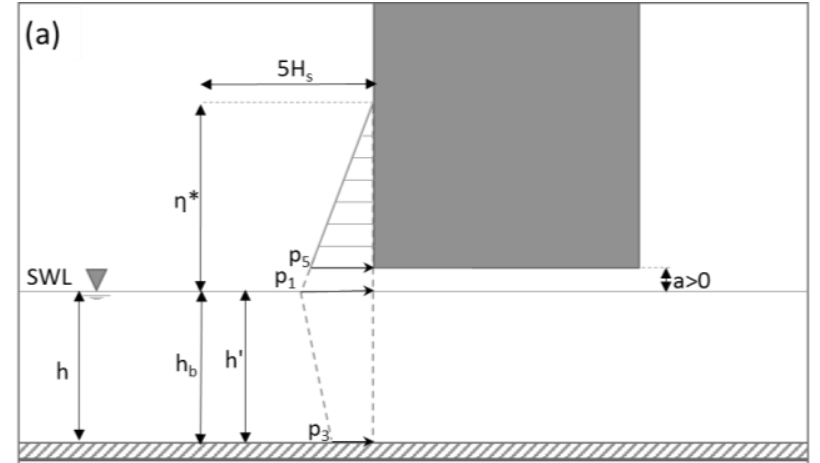

(b)

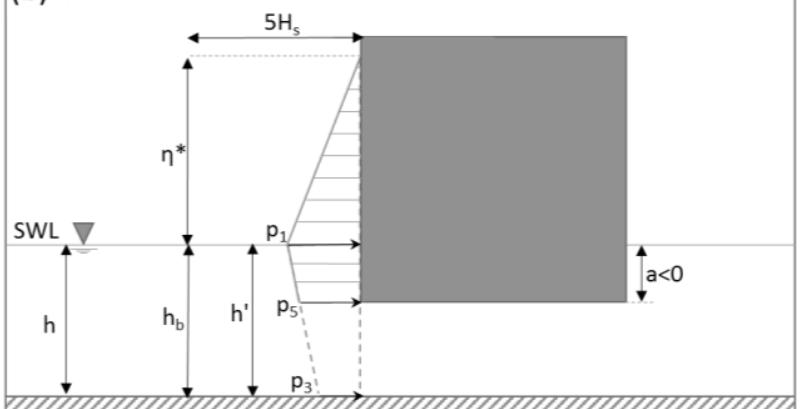

Figure 3: Goda's (2010) pressure distribution for a vertical caisson; modified by Wiebe et al. (2014) for structural air gaps above (a) and below (b) the stillwater level.

A vertical force equation was developed based on experimental results and validated for regular and irregular wave trials. The equation used readily calculated values obtained from the Wiebe (2014) modifications to the Goda (2010) wave pressure equations to estimate the average pressure on the base of the structure at the time of the maximum vertical force.

\section{RESULTS AND MODIFICATIONS FOR ELEVATED STRUCTURES}

Results indicated that the horizontal pressure distribution associated with a nonbreaking wave was generally consistent when the structure's position above or below the still water level was varied. This consistency is observed in Fig. 4, which superimposes the pressures measured at their respective depths for all trials of the same wave condition.
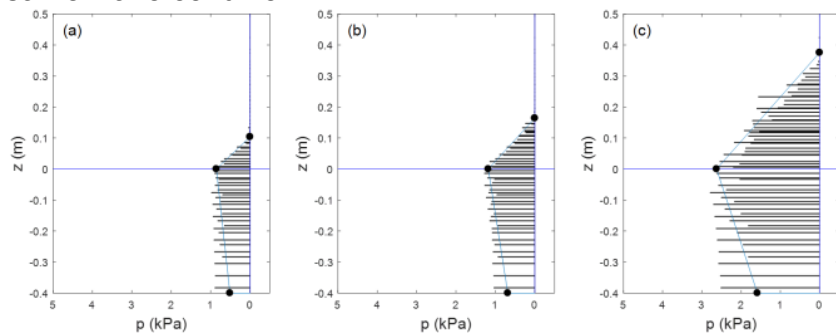

Figure 4: Vertical pressure distributions for regular waves with (a) $\mathrm{X} 1, \mathrm{H}^{-}=0.10 \mathrm{~m}$, (b) $\mathrm{X} 6, \mathrm{H}^{-}=0.15 \mathrm{~m}$, and (c) $\mathrm{X} 2, \mathrm{H}^{-}=0.23$ $\mathrm{m}$, based on all air gap trials for a given wave condition. Averaged values of $p 1, p 3$, and $\eta^{*}$ are marked as filled black circles.

Comparisons of the measured force obtained by integrating pressure sensors over the vertical domain of the structure and the predicted force obtained by integrating the pressure distributions given by the Goda
(2010) equations for wave-induced pressures on a caisson breakwater indicated reasonable agreement for measured average (regular) and significant (irregular) nonimpulsive wave forces. Predictions were generally conservative by a factor of 1.22 for regular wave heights. Considering irregular wave trials, the Goda (2010) equations produced horizontal forces that agreed well with measured forces for smaller considered wave heights (e.g. $H_{1 / 3}$ ), and predictions became conservative when considering extreme wave heights (e.g. $H_{1 / 50)}$ that may have approached breaking conditions. Impulsive wave pressures were not included in the Goda (2010) approximation and therefore coefficients proposed by Takahashi et al. (1994) may result in better agreement for these conditions.

A simple empirical equation to estimate the vertical force as a uniform distribution with average pressure equal to 0.54 times the pressure at the base of the structure at the time of the maximum horizontal force showed reasonable agreement with the integrated measured vertical force for both regular waves and the significant force $\left(F_{V}, 1 / 3\right)$ for irregular waves. Scale factors were applied to account for consideration of larger significant forces (e.g. $F_{V, 1 / 10}$, $F_{V, 1 / 50)}$. Additional work is required to characterize the effects of the wave length and bottom area on the total uplift force associated with nonbreaking waves.

\section{CONCLUSIONS}

This work represents one of the first large scale, detailed investigations of wave-induced pressure distributions on elevated structures. Significant results indicate that the horizontal pressure distribution can be characterized along its vertical extent regardless of a structure's position above or below the stillwater level. The equations presented by Goda (2010) and modified by Wiebe et al. (2014) reasonably estimated the total horizontal force due to regular waves and the significant force due to irregular waves. Finally, this work extends previous studies to investigate 3D effects of wavestructure interaction including horizontal and vertical wave-induced forces. Additional work is necessary to characterize the effect of impulsive wave impacts on coastal structures. This work based on data collected during the experiment described by Lomonaco et al. (2018).

\section{REFERENCES}

Goda (2010): Random seas and design of maritime structures. $3^{\text {rd }}$ edition. S.1.: World Scientific.

Takahashi, S., Tanimoto, K., and Simosako, K. 1994. A proposal of impulsive pressure coefficient for the design of composite breakwaters. Proc. Int. Conf. HydroTechnical Engrg. for Port and Harbor Constr. (Hydro-Port 94), Yokosuka, Japan, 489-504.

Wiebe, Park., and Cox (2014): Application of the Goda pressure formulae for horizontal wave loads on elevated structures. KSCE J. Civil Eng. vol.18 (6), pp. 1573-1579. 